\title{
An intermixed algorithm for strict pseudo-contractions in Hilbert spaces
}

\author{
Zhangsong Yao ${ }^{1}$, Shin Min Kang ${ }^{2 *}$ and Hong-Jun $\mathrm{Li}^{3}$
}

\author{
"Correspondence: \\ smkang@gnu.ac.kr \\ 2Department of Mathematics and \\ the RINS, Gyeongsang National \\ University, Jinju, 660-701, Korea \\ Full list of author information is \\ available at the end of the article
}

\begin{abstract}
An intermixed algorithm for two strict pseudo-contractions in Hilbert spaces have been presented. It is shown that the suggested algorithms converge strongly to the fixed points of two strict pseudo-contractions, independently. As a special case, we can find the common fixed points of two strict pseudo-contractions in Hilbert spaces.
\end{abstract}

MSC: $47 \mathrm{H} 09 ; 47 \mathrm{H} 10$

Keywords: intermixed algorithm; strict pseudo-contraction; fixed point; strong convergence

\section{Introduction}

Let $C$ be a nonempty closed convex subset of a real Hilbert space $H$ with its inner product $\langle\cdot, \cdot\rangle$ and norm $\|\cdot\|$.

Definition 1.1 A mapping $T: C \rightarrow C$ is said to be nonexpansive if

$$
\|T x-T y\| \leq\|x-y\|
$$

for all $x, y \in C$.

We use $\operatorname{Fix}(T)$ to denote the set of fixed points of $T$.

Definition 1.2 A mapping $T: C \rightarrow C$ is said to be strictly pseudo-contractive if there exists a constant $0 \leq \lambda<1$ such that

$$
\|T x-T y\|^{2} \leq\|x-y\|^{2}+\lambda\|(I-T) x-(I-T) y\|^{2}, \quad \forall x, y \in C .
$$

Remark 1.3 It is well known that the class of strictly pseudo-contractive mappings properly includes the class of nonexpansive mappings.

Iterative construction of fixed points of nonlinear mappings has a long history and is still an active field in the nonlinear functional analysis. Let $C$ be a nonempty closed convex subset of a real Hilbert space. Let $T: C \rightarrow C$ be a nonlinear mapping. Let $\left\{\alpha_{n}\right\}$ be a real number sequence in $(0,1)$. For arbitrarily fixed $x_{0} \in C$, define a sequence $\left\{x_{n}\right\}$ in the

(c) 2015 Yao et al. This article is distributed under the terms of the Creative Commons Attribution 4.0 International License (http://creativecommons.org/licenses/by/4.0/), which permits unrestricted use, distribution, and reproduction in any medium, provided you give appropriate credit to the original author(s) and the source, provide a link to the Creative Commons license, and indicate if changes were made. 
following manner:

$$
x_{n+1}=\alpha_{n} x_{n}+\left(1-\alpha_{n}\right) T x_{n}, \quad n \geq 0 .
$$

Iteration (1.1) is said to be a Mann iteration [1]; it has been studied extensively in the literature. If $T$ is a nonexpansive mapping with $\operatorname{Fix}(T) \neq \emptyset$ and $\left\{\alpha_{n}\right\}$ satisfies the condition $\sum_{n=0}^{\infty} \alpha_{n}\left(1-\alpha_{n}\right)=\infty$, then the sequence $\left\{x_{n}\right\}$ generated by Mann's algorithm converges weakly to a fixed point of $T$ [2]. Now, it is well known that Mann's algorithm fails, in general, to converge strongly in the setting of infinite-dimensional Hilbert spaces [3]. Iterative methods for nonexpansive mappings have been investigated extensively in the literature; see [2-27] and the references therein. However, iterative methods for strictly pseudo-contractive mappings are far less developed than those for nonexpansive mappings though Browder and Petryshyn [4] initiated their work in 1967. However, strictly pseudo-contractive mappings have more powerful applications than nonexpansive mappings, for example, to solve inverse problems (see Scherzer [21]). Therefore it is interesting to develop the algorithms for finding the fixed points of strictly pseudo-contractive mappings. Now, we know that Mann's algorithm is not good enough for approximating fixed points of (even if Lipschitz continuous) pseudo-contractions. Thus, we have to find other type of iterative algorithms; see [28-35]. The first such an attempt was done by Ishikawa [7] who introduced the following Ishikawa algorithm:

$$
\begin{aligned}
& y_{n}=\left(1-\beta_{n}\right) x_{n}+\beta_{n} T x_{n}, \\
& x_{n+1}=\left(1-\alpha_{n}\right) x_{n}+\alpha_{n} T y_{n},
\end{aligned}
$$

where $\left\{\alpha_{n}\right\}$ and $\left\{\beta_{n}\right\}$ are sequences in the interval $[0,1], T$ is a (nonlinear) self-mapping of $C$, and the initial guess $x_{0} \in C$ is selected arbitrarily. (Ishikawa's algorithm can be viewed as a double-step (or two-level) Mann's algorithm.) Ishikawa proved that his algorithm converges in norm to a fixed point of a Lipschitz pseudo-contraction $T$ if $\left\{\alpha_{n}\right\}$ and $\left\{\beta_{n}\right\}$ satisfy certain conditions and if $T$ is compact.

On the other hand, iterative methods for approximating the common fixed points of a finite (or an infinite) family of nonlinear mappings have been considered by many authors. For the related work, we refer the reader to [22-26, 32, 33]. Above discussion suggests the following question.

Question 1.4 Could we construct an iterative algorithm such that it converges strongly to the fixed points of a finite family of strict pseudo-contractions?

It is our purpose in this paper to construct redundant intermixed algorithms for two strict pseudo-contractions. It is shown that the suggested algorithms converge strongly to the fixed points of two strict pseudo-contractions, independently. As a special case, we can find the common fixed points of two strict pseudo-contractions in Hilbert spaces.

\section{Preliminaries}

Let $C$ be a nonempty closed convex subset of $H$. The (nearest point or metric) projection from $H$ onto $C$ is defined as follows: for each point $x \in H, P_{C} x$ is the unique point in $C$ 
with the property:

$$
\left\|x-P_{C} x\right\| \leq\|x-y\|, \quad y \in C .
$$

Note that $P_{C}$ is characterized by the inequality:

$$
P_{C} x \in C, \quad\left\langle x-P_{C} x, y-P_{C} x\right\rangle \leq 0, \quad y \in C .
$$

Consequently, $P_{C}$ is nonexpansive.

In order to prove our main results, we need the following well-known lemmas.

Lemma 2.1 ([28]) Let C be a nonempty closed convex subset of a real Hilbert space H. Let $T: C \rightarrow C$ be a $\lambda$-strictly pseudo-contractive mapping. Then $I-T$ is demi-closed at 0 , i.e., if $x_{n} \rightarrow x \in C$ and $x_{n}-T x_{n} \rightarrow 0$, then $x=T x$.

Lemma 2.2 ([18]) Let $\left\{x_{n}\right\}$ and $\left\{y_{n}\right\}$ be bounded sequences in a Banach space $E$ and $\left\{\beta_{n}\right\}$ be a sequence in $[0,1]$ with $0<\liminf _{n \rightarrow \infty} \beta_{n} \leq \lim \sup _{n \rightarrow \infty} \beta_{n}<1$. Suppose that $x_{n+1}=(1-$ $\left.\beta_{n}\right) x_{n}+\beta_{n} z_{n}$ for all $n \geq 0$ and $\lim _{\sup } \rightarrow \infty\left(\left\|z_{n+1}-z_{n}\right\|-\left\|x_{n+1}-x_{n}\right\|\right) \leq 0$. Then $\lim _{n \rightarrow \infty} \| z_{n}-$ $x_{n} \|=0$.

Lemma 2.3 ([17]) Assume $\left\{a_{n}\right\}$ is a sequence of nonnegative real numbers such that $a_{n+1} \leq$ $\left(1-\gamma_{n}\right) a_{n}+\gamma_{n} \delta_{n}, n \geq 0$ where $\left\{\gamma_{n}\right\}$ is a sequence in $(0,1)$ and $\left\{\delta_{n}\right\}$ is a sequence in $R$ such that

(i) $\sum_{n=0}^{\infty} \gamma_{n}=\infty$;

(ii) $\lim _{\sup } \operatorname{sum}_{n \rightarrow \infty} \delta_{n} \leq 0$ or $\sum_{n=0}^{\infty}\left|\delta_{n} \gamma_{n}\right|<\infty$.

Then $\lim _{n \rightarrow \infty} a_{n}=0$.

\section{Main results}

Let $C$ be a nonempty closed convex subset of a real Hilbert space $H$. Let $T: C \rightarrow C$ be a $\lambda$-strict pseudo-contraction. Let $f: C \rightarrow H$ be a $\rho_{1}$-contraction and $g: C \rightarrow H$ be a $\rho_{2}$-contraction. (A mapping $f: C \rightarrow H$ is said to be contractive if $\|f(x)-f(y)\| \leq \rho\|x-y\|$ for some $\rho \in[0,1)$ and for all $x, y \in C$.) Let $k \in(0,1-\lambda)$ be a constant.

Now we propose the following redundant intermixed algorithm for two strict pseudocontractions $S$ and $T$.

Algorithm 3.1 For arbitrarily given $x_{0} \in C, y_{0} \in C$, let the sequences $\left\{x_{n}\right\}$ and $\left\{y_{n}\right\}$ be generated iteratively by

$$
\begin{cases}x_{n+1}=\left(1-\beta_{n}\right) x_{n}+\beta_{n} P_{C}\left[\alpha_{n} f\left(y_{n}\right)+\left(1-k-\alpha_{n}\right) x_{n}+k T x_{n}\right], & n \geq 0, \\ y_{n+1}=\left(1-\beta_{n}\right) y_{n}+\beta_{n} P_{C}\left[\alpha_{n} g\left(x_{n}\right)+\left(1-k-\alpha_{n}\right) y_{n}+k S y_{n}\right], & n \geq 0,\end{cases}
$$

where $\left\{\alpha_{n}\right\}$ and $\left\{\beta_{n}\right\}$ are two real number sequences in $(0,1)$.

Remark 3.2 Note that the definition of the sequence $\left\{x_{n}\right\}$ is involved in the sequence $\left\{y_{n}\right\}$ and the definition of the sequence $\left\{y_{n}\right\}$ is also involved in the sequence $\left\{x_{n}\right\}$. So, this algorithm is said to be the redundant intermixed algorithm. We can use this algorithm to find the fixed points of $S$ and $T$, independently. 
Theorem 3.3 Suppose that $\operatorname{Fix}(S) \neq \emptyset$ and $\operatorname{Fix}(T) \neq \emptyset$. Assume the following conditions are satisfied:

(C1) $\lim _{n \rightarrow \infty} \alpha_{n}=0$ and $\sum_{n=0}^{\infty} \alpha_{n}=\infty$;

(C2) $\beta_{n} \in\left[\xi_{1}, \xi_{2}\right] \subset(0,1)$ for all $n \geq 0$.

Then the sequences $\left\{x_{n}\right\}$ and $\left\{y_{n}\right\}$ generated by (3.1) converge strongly to the fixed points $P_{\mathrm{Fix}(T)} f\left(y^{*}\right)$ and $P_{\mathrm{Fix}(S)} g\left(x^{*}\right)$ of $T$ and $S$, respectively, where $x^{*} \in \operatorname{Fix}(T)$ and $y^{*} \in \operatorname{Fix}(S)$.

Proof First, we give the following propositions.

Proposition 3.4 The sequences $\left\{x_{n}\right\}$ and $\left\{y_{n}\right\}$ are bounded.

In order to prove this proposition, we need the following result.

Proposition 3.5 The mapping $P_{C}[\alpha f+(1-k-\alpha) I+k T]$ is contractive for small enough $\alpha$.

Proof Let $x, y \in C$. Then we have

$$
\begin{aligned}
\left\|P_{C}[\alpha f(x)+(1-k-\alpha) x+k T x]-P_{C}[\alpha f(y)+(1-k-\alpha) y+k T y]\right\|^{2} \\
\leq\|\alpha(f(x)-f(y))+(1-k-\alpha)(x-y)+k(T x-T y)\|^{2} \\
=\left\|\alpha(f(x)-f(y))+(1-\alpha)\left[\frac{1-k-\alpha}{1-\alpha}(x-y)+\frac{k}{1-\alpha}(T x-T y)\right]\right\|^{2} \\
\leq \alpha\|f(x)-f(y)\|^{2}+(1-\alpha)\left\|\frac{1-k-\alpha}{1-\alpha}(x-y)+\frac{k}{1-\alpha}(T x-T y)\right\|^{2} \\
\leq \alpha \rho_{1}\|x-y\|^{2}+\frac{(1-k-\alpha)^{2}}{1-\alpha}\|x-y\|^{2}+\frac{k^{2}}{1-\alpha}\|T x-T y\|^{2} \\
\quad+\frac{2(1-k-\alpha) k}{1-\alpha}\langle T x-T y, x-y\rangle \\
\leq \alpha \rho_{1}\|x-y\|^{2}+\frac{(1-k-\alpha)^{2}}{1-\alpha}\|x-y\|^{2}+\frac{k^{2}}{1-\alpha}\left[\|x-y\|^{2}+\lambda\|(I-T) x-(I-T) y\|^{2}\right] \\
\quad+\frac{2(1-k-\alpha) k}{1-\alpha}\left[\|x-y\|^{2}-\frac{1-\lambda}{2}\|(I-T) x-(I-T) y\|^{2}\right] \\
=\alpha \rho_{1}\|x-y\|^{2}+\frac{1}{1-\alpha}\left[\lambda k^{2}-(1-\lambda)(1-k-\alpha) k\right]\|(I-T) x-(I-T) y\|^{2} \\
\quad+(1-\alpha)\|x-y\|^{2} \\
=\frac{k}{1-\alpha}[k-(1-\alpha)(1-\lambda)]\|(I-T) x-(I-T) y\|^{2}+\left[1-\left(1-\rho_{1}\right) \alpha\right]\|x-y\|^{2} .
\end{aligned}
$$

Thus, we get

$$
\begin{aligned}
& \left\|P_{C}[\alpha f(x)+(1-k-\alpha) x+k T x]-P_{C}[\alpha f(y)+(1-k-\alpha) y+k T y]\right\| \\
& \quad \leq\left[1-\frac{\left(1-\rho_{1}\right) \alpha}{2}\right]\|x-y\|
\end{aligned}
$$

for all $x, y \in C$ as $k \leq(1-\alpha)(1-\lambda)$ (that is, $\left.\alpha \leq 1-\frac{k}{1-\lambda}\right)$.

Next, we prove Proposition 3.4. 
Proof Since $\operatorname{Fix}(S) \neq \emptyset$ and $\operatorname{Fix}(T) \neq \emptyset$, we can choose $x^{*} \in \operatorname{Fix}(T)$ and $y^{*} \in \operatorname{Fix}(S)$. From (3.1), we have

$$
\begin{aligned}
\left\|x_{n+1}-x^{*}\right\|= & \left\|\left(1-\beta_{n}\right) x_{n}+\beta_{n} P_{C}\left[\alpha_{n} f\left(y_{n}\right)+\left(1-k-\alpha_{n}\right) x_{n}+k T x_{n}\right]-x^{*}\right\| \\
\leq & \beta_{n}\left\|P_{C}\left[\alpha_{n} f\left(y_{n}\right)+\left(1-k-\alpha_{n}\right) x_{n}+k T x_{n}\right]-x^{*}\right\| \\
& +\left(1-\beta_{n}\right)\left\|x_{n}-x^{*}\right\| \\
\leq & \beta_{n} \alpha_{n}\left\|f\left(y_{n}\right)-x^{*}\right\|+\beta_{n}\left\|\left(1-k-\alpha_{n}\right)\left(x_{n}-x^{*}\right)+k\left(T x_{n}-T x^{*}\right)\right\| \\
& +\left(1-\beta_{n}\right)\left\|x_{n}-x^{*}\right\| \\
\leq & \beta_{n} \alpha_{n}\left\|f\left(y_{n}\right)-f\left(y^{*}\right)\right\|+\beta_{n} \alpha_{n}\left\|f\left(y^{*}\right)-x^{*}\right\|+\left(1-\beta_{n}\right)\left\|x_{n}-x^{*}\right\| \\
& +\beta_{n}\left(1-\alpha_{n}\right)\left\|x_{n}-x^{*}\right\| \\
\leq & \rho_{1} \beta_{n} \alpha_{n}\left\|y_{n}-y^{*}\right\|+\beta_{n} \alpha_{n}\left\|f\left(y^{*}\right)-x^{*}\right\|+\left(1-\alpha_{n} \beta_{n}\right)\left\|x_{n}-x^{*}\right\| \\
\leq & \rho \beta_{n} \alpha_{n}\left\|y_{n}-y^{*}\right\|+\beta_{n} \alpha_{n}\left\|f\left(y^{*}\right)-x^{*}\right\|+\left(1-\alpha_{n} \beta_{n}\right)\left\|x_{n}-x^{*}\right\|,
\end{aligned}
$$

where $\rho=\max \left\{\rho_{1}, \rho_{2}\right\}$. Similarly, we have

$$
\begin{aligned}
\left\|y_{n+1}-y^{*}\right\| & \leq \rho_{2} \beta_{n} \alpha_{n}\left\|x_{n}-x^{*}\right\|+\beta_{n} \alpha_{n}\left\|g\left(x^{*}\right)-y^{*}\right\|+\left(1-\alpha_{n} \beta_{n}\right)\left\|y_{n}-y^{*}\right\| \\
& \leq \rho \beta_{n} \alpha_{n}\left\|x_{n}-x^{*}\right\|+\beta_{n} \alpha_{n}\left\|g\left(x^{*}\right)-y^{*}\right\|+\left(1-\alpha_{n} \beta_{n}\right)\left\|y_{n}-y^{*}\right\| .
\end{aligned}
$$

Hence, we obtain

$$
\begin{aligned}
& \left\|x_{n+1}-x^{*}\right\|+\left\|y_{n+1}-y^{*}\right\| \\
& \quad \leq\left[1-(1-\rho) \alpha_{n} \beta_{n}\right]\left(\left\|x_{n}-x^{*}\right\|+\left\|y_{n}-y^{*}\right\|\right)+\alpha_{n} \beta_{n}\left(\left\|f\left(y^{*}\right)-x^{*}\right\|+\left\|g\left(x^{*}\right)-y^{*}\right\|\right) \\
& \quad \leq \max \left\{\left\|x_{n}-x^{*}\right\|+\left\|y_{n}-y^{*}\right\|, \frac{\left\|f\left(y^{*}\right)-x^{*}\right\|+\left\|g\left(x^{*}\right)-y^{*}\right\|}{1-\rho}\right\} .
\end{aligned}
$$

By induction, we have

$$
\begin{aligned}
& \left\|x_{n}-x^{*}\right\|+\left\|y_{n}-y^{*}\right\| \\
& \quad \leq \max \left\{\left\|x_{0}-x^{*}\right\|+\left\|y_{0}-y^{*}\right\|, \frac{\left\|f\left(y^{*}\right)-x^{*}\right\|+\left\|g\left(x^{*}\right)-y^{*}\right\|}{1-\alpha}\right\} .
\end{aligned}
$$

So, $\left\{x_{n}\right\}$ and $\left\{y_{n}\right\}$ are bounded.

Proposition 3.6 $\left\|x_{n}-T x_{n}\right\| \rightarrow 0$ and $\left\|y_{n}-S y_{n}\right\| \rightarrow 0$.

Proof We first estimate $\left\|x_{n+1}-x_{n}\right\|$. Set $u_{n}=P_{C}\left[\alpha_{n} f\left(y_{n}\right)+\left(1-k-\alpha_{n}\right) x_{n}+k T x_{n}\right], n \geq 0$. It follows that

$$
\begin{aligned}
\left\|u_{n+1}-u_{n}\right\| \leq & \| \alpha_{n+1} f\left(y_{n+1}\right)+\left(1-k-\alpha_{n+1}\right) x_{n+1}+k T x_{n+1} \\
& -\alpha_{n} f\left(y_{n}\right)-\left(1-k-\alpha_{n}\right) x_{n}+k T x_{n} \| \\
\leq & \left\|\left(1-k-\alpha_{n+1}\right)\left(x_{n+1}-x_{n}\right)+k\left(T x_{n+1}-T x_{n}\right)\right\|
\end{aligned}
$$




$$
\begin{aligned}
& +\alpha_{n+1}\left(\left\|f\left(y_{n+1}\right)\right\|+\left\|x_{n}\right\|\right)+\alpha_{n}\left(\left\|f\left(y_{n}\right)\right\|+\left\|x_{n}\right\|\right) \\
\leq & \left(1-\alpha_{n+1}\right)\left\|x_{n+1}-x_{n}\right\|+\alpha_{n+1}\left(\left\|f\left(y_{n+1}\right)\right\|+\left\|x_{n}\right\|\right) \\
& +\alpha_{n}\left(\left\|f\left(y_{n}\right)\right\|+\left\|x_{n}\right\|\right) .
\end{aligned}
$$

Since $\alpha_{n} \rightarrow 0$, we deduce that

$$
\limsup _{n \rightarrow \infty}\left(\left\|u_{n+1}-u_{n}\right\|-\left\|x_{n+1}-x_{n}\right\|\right) \leq 0 .
$$

From Lemma 2.2, we get

$$
\lim _{n \rightarrow \infty}\left\|u_{n}-x_{n}\right\|=0 \quad \text { and } \quad \lim _{n \rightarrow \infty}\left\|x_{n+1}-x_{n}\right\|=0
$$

From (3.1), we derive

$$
\begin{aligned}
\left\|x_{n+1}-T x_{n}\right\| \leq & \left(1-\beta_{n}\right)\left\|x_{n}-T x_{n}\right\|+\beta_{n} \alpha_{n}\left\|f\left(y_{n}\right)-T x_{n}\right\| \\
& +\beta_{n}\left(1-k-\alpha_{n}\right)\left\|x_{n}-T x_{n}\right\| \\
= & {\left[1-\left(k+\alpha_{n}\right) \beta_{n}\right]\left\|x_{n}-T x_{n}\right\|+\beta_{n} \alpha_{n}\left\|f\left(y_{n}\right)-T x_{n}\right\| . }
\end{aligned}
$$

Thus,

$$
\begin{aligned}
\left\|x_{n}-T x_{n}\right\| \leq & \left\|x_{n}-x_{n+1}\right\|+\left\|x_{n+1}-T x_{n}\right\| \\
\leq & {\left[1-\left(k+\alpha_{n}\right) \beta_{n}\right]\left\|x_{n}-T x_{n}\right\|+\beta_{n} \alpha_{n}\left\|f\left(y_{n}\right)-T x_{n}\right\| } \\
& +\left\|x_{n}-x_{n+1}\right\| .
\end{aligned}
$$

It follows that

$$
\begin{aligned}
\left\|x_{n}-T x_{n}\right\| & \leq \frac{1}{\left(k+\alpha_{n}\right) \beta_{n}}\left(\left\|x_{n}-x_{n+1}\right\|+\beta_{n} \alpha_{n}\left\|f\left(y_{n}\right)-T x_{n}\right\|\right) \\
& \rightarrow 0 .
\end{aligned}
$$

Similarly, we can obtain

$$
\lim _{n \rightarrow \infty}\left\|y_{n}-S y_{n}\right\|=0 .
$$

By Proposition 3.5, we know that the mapping $P_{C}[\alpha f+(1-k-\alpha) I+k T]$ is contractive for small enough $\alpha$. Thus, the equation $x=P_{C}[t f(x)+(1-k-t) x+k T x]$ has a unique fixed point, denoted by $x_{t}$, that is,

$$
x_{t}=P_{C}\left[t f\left(x_{t}\right)+(1-k-t) x_{t}+k T x_{t}\right]
$$

for small enough $t$. In order to prove Theorem 3.3, we need the following lemma.

Lemma 3.7 Suppose $\operatorname{Fix}(T) \neq \emptyset$. Then, as $t \rightarrow 0$, the net $\left\{x_{t}\right\}$ defined by (3.2) converges strongly to a fixed point of $T$. 
Proof Let $x^{*} \in \operatorname{Fix}(T)$. From (3.2), we have

$$
\begin{aligned}
\left\|x_{t}-x^{*}\right\| & =\left\|P_{C}\left[t f\left(x_{t}\right)+(1-k-t) x_{t}+k T x_{t}\right]-x^{*}\right\| \\
& \leq t\left\|f\left(x_{t}\right)-x^{*}\right\|+\left\|(1-k-t)\left(x_{t}-x^{*}\right)+k\left(T x_{t}-x^{*}\right)\right\| \\
& \leq t \rho_{1}\left\|x_{t}-x^{*}\right\|+t\left\|f\left(x^{*}\right)-x^{*}\right\|+(1-t)\left\|x_{t}-x^{*}\right\|,
\end{aligned}
$$

hence,

$$
\left\|x_{t}-x^{*}\right\| \leq \frac{1}{1-\rho_{1}}\left\|f\left(x^{*}\right)-x^{*}\right\| .
$$

Thus, $\left\{x_{t}\right\}$ is bounded. Again, from (3.2), we get

$$
\left\|x_{t}-T x_{t}\right\| \leq t\left\|f\left(x_{t}\right)-T x_{t}\right\|+(1-k-t)\left\|x_{t}-T x_{t}\right\| .
$$

It follows that

$$
\left\|x_{t}-T x_{t}\right\| \leq \frac{t}{k+t}\left\|f\left(x_{t}\right)-T x_{t}\right\| \rightarrow 0 .
$$

Let $\left\{t_{n}\right\} \subset(0,1)$. Assume that $t_{n} \rightarrow 0$ as $n \rightarrow \infty$. Put $x_{n}:=x_{t_{n}}$. We have $\lim _{n \rightarrow \infty} \| x_{n}-$ $T x_{n} \|=0$. Set $y_{t}=t f\left(x_{t}\right)+(1-k-t) x_{t}+k T x_{t}$, for all $t$. Then we have $x_{t}=P_{C} y_{t}$, and for any $x^{*} \in \operatorname{Fix}(T)$,

$$
\begin{aligned}
x_{t}-x^{*} & =x_{t}-y_{t}+y_{t}-x^{*} \\
& =x_{t}-y_{t}+t\left(f\left(x_{t}\right)-x^{*}\right)+(1-k-t)\left(x_{t}-x^{*}\right)+k\left(T x_{t}-x^{*}\right) .
\end{aligned}
$$

From the property of the metric projection, we deduce

$$
\left\langle x_{t}-y_{t}, x_{t}-x^{*}\right\rangle \leq 0 \text {. }
$$

So,

$$
\begin{aligned}
\left\|x_{t}-x^{*}\right\|^{2}= & \left\langle x_{t}-y_{t}, x_{t}-x^{*}\right\rangle+\left\langle(1-k-t)\left(x_{t}-x^{*}\right)+k\left(T x_{t}-x^{*}\right), x_{t}-x^{*}\right\rangle \\
& +t\left|f\left(x_{t}\right)-x^{*}, x_{t}-x^{*}\right\rangle \\
\leq & \left\|(1-k-t)\left(x_{t}-x^{*}\right)+k\left(T x_{t}-x^{*}\right)\right\|\left\|x_{t}-x^{*}\right\| \\
& \left.\quad+t f f\left(x_{t}\right)-f\left(x^{*}\right), x_{t}-x^{*}\right\rangle+t\left|f\left(x^{*}\right)-x^{*}, x_{t}-x^{*}\right\rangle \\
\leq & {\left[1-\left(1-\rho_{1}\right) t\right]\left\|x_{t}-x^{*}\right\|^{2}+t\left\langle f\left(x^{*}\right)-x^{*}, x_{t}-x^{*}\right\rangle . }
\end{aligned}
$$

Hence,

$$
\left\|x_{t}-x^{*}\right\|^{2} \leq \frac{1}{\left(1-\rho_{1}\right)}\left\{f\left(x^{*}\right)-x^{*}, x_{t}-x^{*}\right), \quad \forall x^{*} \in \operatorname{Fix}(T) .
$$

By similar arguments to [28], we find that the net $\left\{x_{t}\right\}$ converges strongly to $x^{*} \in \operatorname{Fix}(T)$. This completes the proof. 
Remark 3.8 From Lemma 3.7, we know that the net $\left\{x_{t}\right\}$ defined by $x_{t}=P_{C}[t u+(1-k-$ $\left.t) x_{t}+k T x_{t}\right]$ where $u \in H$, converges to $P_{\mathrm{Fix}(T)} u$. Let $x^{*} \in \operatorname{Fix}(T)$ and $y^{*} \in \operatorname{Fix}(S)$. If we take $u=f\left(y^{*}\right)$, then the net $\left\{x_{t}\right\}$ defined by $x_{t}=P_{C}\left[t f\left(y^{*}\right)+(1-k-t) x_{t}+k T x_{t}\right]$, converges to $P_{\operatorname{Fix}(T)} f\left(y^{*}\right)$.

Finally, we prove that $x_{n} \rightarrow P_{\mathrm{Fix}(T)} f\left(y^{*}\right)$ and $y_{n} \rightarrow P_{\mathrm{Fix}(S)} g\left(x^{*}\right)$, where $x^{*} \in \operatorname{Fix}(T)$ and $y^{*} \in$ Fix $(S)$. We note the following fact. If the sequence $\left\{w_{n}\right\}$ is bounded and $\left\|w_{n}-T w_{n}\right\| \rightarrow 0$, we easily deduce that

$$
\limsup _{n \rightarrow \infty}\left\langle f\left(P_{\mathrm{Fix}(S)} g\left(x^{*}\right)\right)-P_{\mathrm{Fix}(T)} f\left(y^{*}\right), w_{n}-P_{\mathrm{Fix}(T)} f\left(y^{*}\right)\right\rangle \leq 0 .
$$

Set $v_{n}=P_{C}\left[\alpha_{n} g\left(x_{n}\right)+\left(1-k-\alpha_{n}\right) y_{n}+k S y_{n}\right]$ for all $n \geq 0$. Thus, we deduce that the sequences $\left\{u_{n}\right\}$ and $\left\{v_{n}\right\}$ satisfy: (1) $\left\{u_{n}\right\}$ and $\left\{v_{n}\right\}$ are bounded; (2) $\left\|u_{n}-T u_{n}\right\| \rightarrow 0$ and $\left\|v_{n}-S v_{n}\right\| \rightarrow 0$. Therefore,

$$
\limsup _{n \rightarrow \infty}\left\{f\left(P_{\mathrm{Fix}(S)} g\left(x^{*}\right)\right)-P_{\operatorname{Fix}(T)} f\left(y^{*}\right), u_{n}-P_{\operatorname{Fix}(T)} f\left(y^{*}\right)\right\rangle \leq 0
$$

and

$$
\limsup _{n \rightarrow \infty}\left\langle g\left(P_{\mathrm{Fix}(T)} f\left(y^{*}\right)\right)-P_{\mathrm{Fix}(S)} g\left(x^{*}\right), v_{n}-P_{\mathrm{Fix}(S)} g\left(x^{*}\right)\right\rangle \leq 0 .
$$

Next, we estimate $\left\|u_{n}-P_{\operatorname{Fix}(T)} f\left(y^{*}\right)\right\|$. Set $\tilde{u}_{n}=\alpha_{n} f\left(y_{n}\right)+\left(1-k-\alpha_{n}\right) x_{n}+k T x_{n}$ and $\tilde{v}_{n}=$ $\alpha_{n} g\left(x_{n}\right)+\left(1-k-\alpha_{n}\right) y_{n}+k S y_{n}$ for all $n$. We have

$$
\begin{aligned}
& \left\|u_{n}-P_{\operatorname{Fix}(T)} f\left(y^{*}\right)\right\|^{2} \\
& =\left\|P_{C}\left[\tilde{u}_{n}\right]-P_{\mathrm{Fix}(T)} f\left(y^{*}\right)\right\|^{2} \\
& \leq\left\langle\tilde{u}_{n}-P_{\mathrm{Fix}(T)} f\left(y^{*}\right), u_{n}-P_{\mathrm{Fix}(T)} f\left(y^{*}\right)\right\} \\
& =\left\langle\alpha_{n} f\left(y_{n}\right)+\left(1-k-\alpha_{n}\right) x_{n}+k T x_{n}-P_{\mathrm{Fix}(T)} f\left(y^{*}\right), u_{n}-P_{\mathrm{Fix}(T)} f\left(y^{*}\right)\right\rangle \\
& \leq \alpha_{n}\left\{f\left(y_{n}\right)-P_{\mathrm{Fix}(T)} f\left(y^{*}\right), u_{n}-P_{\mathrm{Fix}(T)} f\left(y^{*}\right)\right\rangle \\
& +\left(1-\alpha_{n}\right)\left\|x_{n}-P_{\mathrm{Fix}(T)} f\left(y^{*}\right)\right\|\left\|u_{n}-P_{\mathrm{Fix}(T)} f\left(y^{*}\right)\right\| \\
& \leq \frac{1-\alpha_{n}}{2}\left\|x_{n}-P_{\operatorname{Fix}(T)} f\left(y^{*}\right)\right\|^{2}+\frac{1}{2}\left\|u_{n}-P_{\operatorname{Fix}(T)} f\left(y^{*}\right)\right\|^{2} \\
& +\alpha_{n}\left\langle f\left(y_{n}\right)-f\left(P_{\mathrm{Fix}(S)} g\left(x^{*}\right)\right), u_{n}-P_{\mathrm{Fix}(T)} f\left(y^{*}\right)\right\rangle \\
& +\alpha_{n}\left\langle f\left(P_{\mathrm{Fix}(S)} g\left(x^{*}\right)\right)-P_{\mathrm{Fix}(T)} f\left(y^{*}\right), u_{n}-P_{\mathrm{Fix}(T)} f\left(y^{*}\right)\right\rangle \\
& \leq \frac{1-\alpha_{n}}{2}\left\|x_{n}-P_{\mathrm{Fix}(T)} f\left(y^{*}\right)\right\|^{2}+\frac{1}{2}\left\|u_{n}-P_{\mathrm{Fix}(T)} f\left(y^{*}\right)\right\|^{2} \\
& +\alpha_{n} \rho\left\|y_{n}-P_{\mathrm{Fix}(S)} g\left(x^{*}\right)\right\|\left\|u_{n}-P_{\mathrm{Fix}(T)} f\left(y^{*}\right)\right\| \\
& +\alpha_{n}\left\langle f\left(P_{\mathrm{Fix}(S)} g\left(x^{*}\right)\right)-P_{\mathrm{Fix}(T)} f\left(y^{*}\right), u_{n}-P_{\mathrm{Fix}(T)} f\left(y^{*}\right)\right\rangle \\
& \leq \frac{1-\alpha_{n}}{2}\left\|x_{n}-P_{\operatorname{Fix}(T)} f\left(y^{*}\right)\right\|^{2}+\frac{1}{2}\left\|u_{n}-P_{\operatorname{Fix}(T)} f\left(y^{*}\right)\right\|^{2} \\
& +\frac{\alpha_{n} \rho}{2}\left(\left\|y_{n}-P_{\mathrm{Fix}(S)} g\left(x^{*}\right)\right\|^{2}+\left\|u_{n}-P_{\mathrm{Fix}(T)} f\left(y^{*}\right)\right\|^{2}\right) \\
& +\alpha_{n}\left\langle f\left(P_{\mathrm{Fix}(S)} g\left(x^{*}\right)\right)-P_{\mathrm{Fix}(T)} f\left(y^{*}\right), u_{n}-P_{\mathrm{Fix}(T)} f\left(y^{*}\right)\right) .
\end{aligned}
$$


It follows that

$$
\begin{aligned}
\| u_{n}- & P_{\mathrm{Fix}(T)} f\left(y^{*}\right) \|^{2} \\
\leq & \frac{1-\alpha_{n}}{1-\alpha_{n} \rho}\left\|x_{n}-P_{\mathrm{Fix}(T)} f\left(y^{*}\right)\right\|^{2}+\frac{\alpha_{n} \rho}{1-\alpha_{n} \rho}\left\|y_{n}-P_{\mathrm{Fix}(S)} g\left(x^{*}\right)\right\|^{2} \\
& \quad+\frac{2 \alpha_{n}}{1-\alpha_{n} \rho}\left\langle f\left(P_{\mathrm{Fix}(S)} g\left(x^{*}\right)\right)-P_{\mathrm{Fix}(T)} f\left(y^{*}\right), u_{n}-P_{\mathrm{Fix}(T)} f\left(y^{*}\right)\right\rangle .
\end{aligned}
$$

Thus,

$$
\begin{aligned}
\| x_{n+1}- & P_{\mathrm{Fix}(T)} f\left(y^{*}\right) \|^{2} \\
\leq & \left(1-\beta_{n}\right)\left\|x_{n}-P_{\mathrm{Fix}(T)} f\left(y^{*}\right)\right\|^{2}+\beta_{n}\left\|u_{n}-P_{\mathrm{Fix}(T)} f\left(y^{*}\right)\right\|^{2} \\
\leq & \left(1-\frac{1-\rho}{1-\alpha_{n} \rho} \alpha_{n} \beta_{n}\right)\left\|x_{n}-P_{\mathrm{Fix}(T)} f\left(y^{*}\right)\right\|^{2}+\frac{\alpha_{n} \beta_{n} \rho}{1-\alpha_{n} \rho}\left\|y_{n}-P_{\mathrm{Fix}(S)} g\left(x^{*}\right)\right\|^{2} \\
& +\frac{2 \alpha_{n} \beta_{n}}{1-\alpha_{n} \rho}\left\langle f\left(P_{\mathrm{Fix}(S)} g\left(x^{*}\right)\right)-P_{\mathrm{Fix}(T)} f\left(y^{*}\right), u_{n}-P_{\mathrm{Fix}(T)} f\left(y^{*}\right)\right\rangle .
\end{aligned}
$$

Similarly, we also have

$$
\begin{aligned}
\| y_{n+1}- & P_{\mathrm{Fix}(S)} g\left(x^{*}\right) \|^{2} \\
\leq & \left(1-\frac{1-\rho}{1-\alpha_{n} \rho} \alpha_{n} \beta_{n}\right)\left\|y_{n}-P_{\mathrm{Fix}(S)} g\left(x^{*}\right)\right\|^{2}+\frac{\alpha_{n} \beta_{n} \rho}{1-\alpha_{n} \rho}\left\|x_{n}-P_{\mathrm{Fix}(T)} f\left(y^{*}\right)\right\|^{2} \\
& +\frac{2 \alpha_{n} \beta_{n}}{1-\alpha_{n} \rho}\left\langle g\left(P_{\mathrm{Fix}(T)} f\left(y^{*}\right)\right)-P_{\mathrm{Fix}(S)} g\left(x^{*}\right), v_{n}-P_{\mathrm{Fix}(S)} g\left(x^{*}\right)\right\rangle .
\end{aligned}
$$

Therefore,

$$
\begin{aligned}
\| x_{n+1} & -P_{\mathrm{Fix}(T)} f\left(y^{*}\right)\left\|^{2}+\right\| y_{n+1}-P_{\mathrm{Fix}(S)} g\left(x^{*}\right) \|^{2} \\
\leq & \left(1-\frac{1-2 \rho}{1-\alpha_{n} \rho} \alpha_{n} \beta_{n}\right)\left(\left\|x_{n}-P_{\mathrm{Fix}(T)} f\left(y^{*}\right)\right\|^{2}+\left\|y_{n}-P_{\mathrm{Fix}(S)} g\left(x^{*}\right)\right\|^{2}\right) \\
& +\frac{2 \alpha_{n} \beta_{n}}{1-\alpha_{n} \rho}\left\langle f\left(P_{\mathrm{Fix}(S)} g\left(x^{*}\right)\right)-P_{\mathrm{Fix}(T)} f\left(y^{*}\right), u_{n}-P_{\mathrm{Fix}(T)} f\left(y^{*}\right)\right\rangle \\
& +\frac{2 \alpha_{n} \beta_{n}}{1-\alpha_{n} \rho}\left\langle g\left(P_{\mathrm{Fix}(T)} f\left(y^{*}\right)\right)-P_{\mathrm{Fix}(S)} g\left(x^{*}\right), v_{n}-P_{\mathrm{Fix}(S)} g\left(x^{*}\right)\right\rangle .
\end{aligned}
$$

We can check that all assumptions of Lemma 2.3 are satisfied. Therefore, $x_{n} \rightarrow$ $P_{\mathrm{Fix}(T)} f\left(y^{*}\right)$ and $y_{n} \rightarrow P_{\mathrm{Fix}(S)} g\left(x^{*}\right)$. This completes the proof.

Algorithm 3.9 For arbitrarily given $x_{0} \in C$, let the sequence $\left\{x_{n}\right\}$ be generated iteratively by

$$
x_{n+1}=\left(1-\beta_{n}\right) x_{n}+\beta_{n} P_{C}\left[\left(1-k-\alpha_{n}\right) x_{n}+k T x_{n}\right], \quad n \geq 0,
$$

where $\left\{\alpha_{n}\right\}$ and $\left\{\beta_{n}\right\}$ are two real number sequences in $(0,1)$.

Theorem 3.10 Suppose $\operatorname{Fix}(T) \neq \emptyset$. Assume the following conditions are satisfied: 
(C1) $\lim _{n \rightarrow \infty} \alpha_{n}=0$ and $\sum_{n=0}^{\infty} \alpha_{n}=\infty$;

(C2) $\beta_{n} \in\left[\xi_{1}, \xi_{2}\right] \subset(0,1)$ for all $n \geq 0$.

Then the sequence $\left\{x_{n}\right\}$ generated by (3.3) converge strongly to the fixed points $P_{\mathrm{Fix}(T)}(0)$, which is the minimum norm element in $\operatorname{Fix}(T)$.

\section{Competing interests}

The authors declare that they have no competing interests.

\section{Authors' contributions}

All authors read and approved the final manuscript.

\section{Author details}

${ }^{1}$ School of Information Engineering, Nanjing Xiaozhuang University, Nanjing, 211171, China. ${ }^{2}$ Department of Mathematics and the RINS, Gyeongsang National University, Jinju, 660-701, Korea. ${ }^{3}$ Department of Mathematics, Tianjin Polytechnic University, Tianjin, 300387, China.

\section{Acknowledgements}

The authors are grateful to the three reviewers for their valuable comments and suggestions. Zhangsong Yao was supported by the Scientific Research Project of Nanjing Xiaozhuang University (2015NXY46).

Received: 29 April 2015 Accepted: 4 November 2015 Published online: 14 November 2015

\section{References}

1. Mann, WR: Mean value methods in iteration. Proc. Am. Math. Soc. 4, 506-510 (1953)

2. Reich, S: Weak convergence theorems for non-expansive mappings in Banach spaces. J. Math. Anal. Appl. 67, 274-276 (1979)

3. Genel, A, Lindenstrauss, J: An example concerning fixed points. Isr. J. Math. 22, 81-86 (1975)

4. Browder, FE, Petryshyn, WV: Construction of fixed points of nonlinear mappings. J. Math. Anal. Appl. 20, 197-228 (1967)

5. Browder, FE: Convergence of approximation to fixed points of nonexpansive nonlinear mappings in Hilbert spaces. Arch. Ration. Mech. Anal. 24, 82-90 (1967)

6. Halpern, B: Fixed points of nonexpansive maps. Bull. Am. Math. Soc. 73, 957-961 (1967)

7. Ishikawa, S: Fixed points by a new iteration method. Proc. Am. Math. Soc. 44, 147-150 (1974)

8. Lions, PL: Approximation de points fixes de contractions. C. R. Acad. Sci., Sér. A-B Paris 284, 1357-1359 (1977)

9. Opial, Z: Weak convergence of the sequence of successive approximations of nonexpansive mappings. Bull. Am. Math. Soc. 73, 595-597 (1967)

10. Wittmann, R: Approximation of fixed points of non-expansive mappings. Arch. Math. 58, 486-491 (1992)

11. Moudafi, A: Viscosity approximation methods for fixed-point problems. J. Math. Anal. Appl. 241, 46-55 (2000)

12. Shioji, N, Takahashi, W: Strong convergence of approximated sequences for nonexpansive mappings in Banach spaces. Proc. Am. Math. Soc. 125, 3641-3645 (1997)

13. Suzuki, T: A sufficient and necessary condition for Halpern-type strong convergence to fixed points of nonexpansive mappings. Proc. Am. Math. Soc. 135, 99-106 (2007)

14. Reich, S, Zaslavski, AJ: Convergence of Krasnoselskii-Mann iterations of nonexpansive operators. Math. Comput. Model. 32, 1423-1431 (2000)

15. Xu, HK: Viscosity approximation methods for nonexpansive mappings. J. Math. Anal. Appl. 298, $279-291$ (2004)

16. Geobel, K, Kirk, WA: Topics in Metric Fixed Point Theory. Cambridge Studies in Advanced Mathematics, vol. 28 Cambridge University Press, Cambridge (1990)

17. Xu, HK: Iterative algorithms for nonlinear operators. J. Lond. Math. Soc. 66, 240-256 (2002)

18. Suzuki, T: Strong convergence theorems for infinite families of nonexpansive mappings in general Banach spaces Fixed Point Theory Appl. 2005, 103-123 (2005)

19. Mainge, PE: Approximation methods for common fixed points of nonexpansive mappings in Hilbert spaces. J. Math. Anal. Appl. 325, 469-479 (2007)

20. Chidume, CE, Chidume, CO: Iterative approximation of fixed points of nonexpansive mappings. J. Math. Anal. Appl. 318, 288-295 (2006)

21. Scherzer, O: Convergence criteria of iterative methods based on Landweber iteration for solving nonlinear problems. J. Math. Anal. Appl. 194, 911-933 (1991)

22. Atsushiba, S, Takahashi, W: Strong convergence theorems for a finite family of nonexpansive mappings and applications. Indian J. Math. 41, 435-453 (1999)

23. Bauschke, $\mathrm{HH}$ : The approximation of fixed points of compositions of nonexpansive mappings in Hilbert spaces. J. Math. Anal. Appl. 202, 150-159 (1996)

24. Ceng, LC, Cubiotti, P, Yao, JC: Strong convergence theorems for finitely many nonexpansive mappings and applications. Nonlinear Anal. 67, 1464-1473 (2007)

25. Chang, SS: Viscosity approximation methods for a finite family of nonexpansive mappings in Banach spaces. J. Math. Anal. Appl. 323, 1402-1416 (2006)

26. Jung, JS: Iterative approaches to common fixed points of nonexpansive mappings in Banach spaces. J. Math. Anal. Appl. 302, 509-520 (2005)

27. Yao, Y, Shahzad, N, Liou, YC: Modified semi-implicit midpoint rule for nonexpansive mappings. Fixed Point Theory Appl. 2015, 166 (2015) 
28. Marino, $G, X u, H K$ : Weak and strong convergence theorems for strict pseudocontractions in Hilbert spaces. J. Math. Anal. Appl. 329, 336-349 (2007)

29. Chidume, CE, Mutangadura, SA: An example on the Mann iteration method for Lipschitz pseudo-contractions. Proc. Am. Math. Soc. 129, 2359-2363 (2001)

30. Yao, Y, Liou, YC, Marino, G: A hybrid algorithm for pseudo-contractive mappings. Nonlinear Anal. 71, 997-5002 (2009)

31. Zhou, $\mathrm{H}$ : Strong convergence of an explicit iterative algorithm for continuous pseudo-contractions in Banach spaces. Nonlinear Anal. 70, 4039-4046 (2009)

32. Guo, W, Choi, M, Cho, YJ: Convergence theorems for continuous pseudocontractive mappings in Banach spaces. J. Inequal. Appl. 2014, 384 (2014)

33. Hussain, N, Ćirić, LB, Cho, YJ, Rafiq, A: On Mann-type iteration method for a family of hemicontractive mappings in Hilbert spaces. J. Inequal. Appl. 2013, 41 (2013)

34. Yao, Y, Liou, YC, Yao, JC: Split common fixed point problem for two quasi-pseudo-contractive operators and its algorithm construction. Fixed Point Theory Appl. 2015, 127 (2015)

35. Yao, Y, Postolache, M, Liou, YC, Yao, Z: Construction algorithms for a class of monotone variational inequalities. Optim Lett. (2015). doi:10.1007/s11590-015-0954-8

Submit your manuscript to a SpringerOpen ${ }^{\circ}$ journal and benefit from:

- Convenient online submission

Rigorous peer review

- Immediate publication on acceptance

- Open access: articles freely available online

- High visibility within the field

- Retaining the copyright to your article 